ARTIGO

\title{
MÉTODOS ALTERNATIVOS AO USO DE ANIMAIS COMO RECURSO DIDÁTICO: UM NOVO PARAGIMA BIOÉTICO PARA O ENSINO DA ZOOLOGIA
}

\author{
ANA LAURA DINIZ FURLAN ${ }^{1}$ \\ ORCID: http://orcid.org/0000-0003-3419-3456 \\ MARTA LUCIANE FISCHER ${ }^{2}$ \\ ORCID: http://orcid.org/0000-0002-1885-0535
}

\begin{abstract}
RESUMO: A progressiva intolerância ao uso de animais como recurso didático conduziu à substituição de demonstrações invasivas, sendo questionada no ensino da zoologia, constituinte da formação de professores de ciências e biologia. Tendo como hipótese a incipiência da temática no meio científico e diferenças no cenário nacional e internacional, objetivou-se mapear a concepção do uso de métodos alternativos no contexto acadêmico e pedagógico. A análise quanti e qualitativa de 600 conteúdos atestou, no panorama internacional, a substituição animal relacionada principalmente à pesquisa biomédica. Nacionalmente, destacou-se no ensino, prioritariamente, alternativas ao uso de cães em aulas de técnica cirúrgica para o curso de Medicina Veterinária, sendo ainda escasso nos cursos de zoologia. Concomitantemente, a análise de 289 planos de aula de Zoologia evidenciou que as alternativas estão incorporadas, sendo mais pronunciadas para animais invertebrados, subsidiando a almejada transposição do pensamento tecnicista e utilitário de professores, alunos e sociedade.
\end{abstract}

Palavras-chave: Bioética, Ética animal, Formação do professor de ciências, Métodos alternativos ao uso animal, Zoologia.

\section{ALTERNATIVE METHODS TO THE USE OF ANIMALS WITH DIDACTIC RESOURCES: A NEW BIOETHIC PARADIGM FOR ZOOLOGY EDUCATION?}

\begin{abstract}
The use of animals as a teaching resource has been progressively unacceptable, leading initially to its replacement in invasive interventions, and it has been questioned its applicability in the teaching of zoology. Having the hypothesis of the incipience of the theme in the scientific environment and differences in the national and international scenario, we objected to map the conception of the use

\footnotetext{
${ }^{1}$ Mestre pelo Programa de Pós-Graduação em Bioética, da Pontifícia Universidade Católica do Paraná. Curitiba, PR, Brasil. <ana.ldf@hotmail.com>

${ }^{2}$ Docente do Curso de Biologia e do Programa de Pós-Graduação em Bioética da Pontifícia Universidade Católica do Paraná. Curitiba, PR, Brasil.<marta.fischer@pucpr.br> 
of alternative methods (AM) in the academic and pedagogical context. The quantitative and qualitative analysis of 600 contents attesting that in the international scenario, the animal substitution was mainly related to biomedical research. In the national context, we found some alternatives for replacing dogs used in classes of surgical technique in the course of Veterinary Medicine, however it is still scarce in zoology classes. Concurrently, the analysis of 289 Zoology class plans showed that the alternative methods is incorporated into teacher's routine, being more pronounced for invertebrate animals, subsidizing the transposition of the technical and utilitarian thinking of teachers, students and society.

Keywords: Bioethics, Animal ethics, Science teacher training, Alternative methods to animal use, Zoology.

\section{MÉTODOS ALTERNATIVOS PARA EL USO DE ANIMALES COMO RECURSO DE ENSEÑANZA: UN NUEVO PARAGÍMIO BIOÉTICO PARA ENSEÑAR ZOOLOGÍA}

RESÚMEN: La intolerancia progresiva al uso de animales como recurso didáctico llevó a la su sustitución en classes invasivas, siendo cuestionada em la enseñanza de la zoología, un componente de la formación de profesores de ciencias y biología. Teniendo como hipótesis la incipiencia del tema en la discusión científica y las diferencias en el escenario nacional e internacional, el objetivo de esta investigación fue mapear la concepción del uso de métodos alternativos en el contexto académico y pedagógico. El análisis cuantitativo y cualitativo de 600 contenidos atestiguó en el panorama internacional la sustitución de animales principalmente relacionada con la investigación biomédica. A nivel nacional se destaco, en la enseñanza, principalmente la sustituciónen el uso de perros en las clases de técnica quirúrgica para el curso de Medicina Veterinaria, siendo aún escasa en zoología. Concomitantemente, el análisis de los 289 planes de lecciones de Zoología mostró que las alternativas se incorporan, siendo más pronunciadas para los animales invertebrados, subsidiando la transposición deseada del pensamiento técnico y utilitario de los maestros, estudiantes y la sociedad.

Palabras clave: Bioética Etica animal, Formación de profesores de ciências, Métodos alternativos para uso animal, Zoologia. 


\section{INTRODUÇÃO}

O questionamento dos limites éticos na utilização dos animais, que serviram como fonte de alimentos, peles e óleos, geração de forças e companhia, teve sua origem no uso como modelo para pesquisa científica e estudos acadêmicos, potencializados após a revolução científica (FISCHER, 2017; MAGALHÃES; ORTÊNCIO-FILHO, 2006). A herança mais contundente desse momento histórico foi a concepção difundida por Descartes de que o animal era um ser destituído de senciência, logo, análogo a uma máquina (FISCHER; FURLAN, 2018). Essa visão superou as evidências da relação evolutiva física e emocional entre homens e animais proposta por Darwin, o posicionamento contrário de importantes acadêmicos como Einstein e Voltaire e a conclamação por Van Rensselaer Potter, na década de 1970 (POTTER, 2016), por uma ética pela sobrevivência dependente da transposição de uma postura antropocêntrica para biocêntrica (FISCHER, 2017).

O princípio dos 3R (RUSSEL; BURCH, 1959) balizou a expectativa pela eliminação do uso do modelo animal (replacement), porém, conquanto não fosse exequível, toda pesquisa deveria prover o menor sofrimento possível, instituindo a obrigação moral pela redução (reduction) do número de animais e pelo refinamento (refinament) das técnicas de manejo e intervenção. Concomitantemente, em 1978, a UNESCO publicou a Declaração Universal dos Direitos dos Animais, da qual o Brasil é signatário (DECLARAÇÃO UNIVERSAL DOS DIREITOS DO ANIMAL, 1978), visando fundamentar a elaboração de parâmetros jurídicos para a utilização e convivência harmoniosa com os animais.

Ao desenvolvimento da área da ética voltada para experimentação animal, acrescida das normalizações legais, somou-se a Lei n 11.794 na proteção jurídica dos animais, representada pelo Brasil ao Código Civil de 1916, incrementado na Constituição Federal de 1988 e consolidada na efetuação das Leis de Crimes Ambientais (9.605/1998)(FISCHER; OLIVEIRA, 2012; BRASIL, 1998, 2008). A Lei Arouca regulamenta, desde 2008, os procedimentos com animais vertebrados submetidos a pesquisas e aulas práticas, sob a supervisão e normatização do Conselho Nacional de Experimentação de Experimentação Animal (CONCEA), através das Diretrizes Brasileiras para o Cuidado e a Utilização de Animais para fins Científicos e Didáticos (DBCA) e supervisão de uma Comissão de Ética no Uso de Animais (CEUA) (CONCEA, 2016; FISCHER; FURLAN, 2018).

O uso de animais em atividades acadêmicas visando a demonstração de processos biológicos conhecidos é tradicionalmente justificado pela sua importância para o aprendizado e legitimado na crença de que os animais não são seres sencientes (FEIJÓ et al., 2010), bem como na confiança atribuída às justificativas acadêmicas (FISCHER; TAMIOSO, 2013). Contudo, o avanço do conhecimento científico, somado à mudança de percepção da sociedade e da comunidade acadêmica, tem gerado repúdio e intolerância à conduta de destituir a vida de seres saudáveis apenas para repetir processos consolidados (BASTOS et al., 2002). Consequentemente, passou a ser considerado crime causar sofrimento ou dor a animais em situações para as quais exista alternativas (CONCEA, 2016). Diante desse novo cenário, paulatinamente o uso de animais em atividades acadêmicas vem sendo, sempre que possível, substituído por manequins, modelos e softwares, partindo-se do pressuposto de que essa substituição não incorra em prejuízo para o aprendizado, contribuindo para a formação de profissionais e cidadãos com condutas éticas e respeito aos animais (DINIZ et al, 2006). Bassalobre (2013), analisando a obra $A$ ética e a formação de professores, de Francisca Severino, reiterou que o educador em formação deve construir instrumentos próprios de intervenção pedagógica, esperando-se que haja uma equalização entre os condicionantes 
culturais e a consciência crítica individual. Assim, espera-se que um professor autônomo, crítico e protagonista, que olhe o animal para além da técnica, seja competente na formação de cidadãos comprometidos com esses novos paradigmas éticos.

A diminuição da utilização do modelo animal nas áreas biomédicas está atrelada ao desenvolvimento de técnicas mais vantajosas e maior adesão dos alunos, baseados no direito à liberdade, assim como maior questionamento acerca do paradoxo em que o educador se insere ao procurar desenvolver posturas éticas, conhecimento e valores e legislações restritivas voltadas especificamente ao uso de animais no ensino (BASTOS et al., 2002). Dentre as aplicações de associação da tecnologia e da ética está a substituição do modelo animal em atividades acadêmicas (FEIJÓ et al., 2010). Tradicionalmente, os animais têm sido utilizados como modelos para experimentação e reconhecimento morfológico. Contudo, as legislações atuais (BRASIL, 1998; 2008) têm normatizado a coleta, captura e experimentação animal demandando o desenvolvimento de métodos alternativos que substitua o uso do animal, mas que não prejudique a capacitação do estudante. Logo, a utilização de tecnologia tornou-se uma meta para as políticas educacionais. Para Levai (2010), o impacto do avanço tecnológico sobre os processos e instituições tem sido significativo e perceptível em vários níveis, porém, propostas de formação de professores parecem caminhar lentamente (LIMA et al., 2008). A complexidade que envolve o aprender exige uma prática docente que repense as estratégias pedagógicas, exigindo competência e condutas éticas (LEVAI, 2010).

Cadáveres de animais são tradicionalmente utilizados em aulas de zoologia, apoiados por educadores que alegam ser impossível desenvolver habilidades necessárias para o exercício profissional do biólogo e do professor de biologia sem contato prévio com o material biológico (FISCHER; FURLAN, 2018). No entanto, para suprir essa demanda se faz necessário coletar espécimes com frequência, uma vez que as dificuldades encontradas para a manutenção de acervos didáticos com manipulação constante requisita reposições de material, principalmente de invertebrados (FISCHER; FURLAN, 2018, MOURA-LEITE; FISCHER, 2018). A retirada dos animais do seu habitat e o possível impacto ecológico gerado por essa atividade, soma-se ao sofrimento causado pelos métodos de coleta, transporte e eutanásia, geralmente realizados de maneira inadequada (FISCHER, 2017; MOURALEIRA; FISCHER, 2018).

Embora existam alternativas formais e informais para uso de animais em aulas de fisiologia, toxicologia, psicologia experimental e anatomia para os cursos de medicina, medicina veterinária e psicologia (SCHANAIDER, 2004, MAGALHÃES; ORTÊNCIO-FILHO, 2006, SEIXAS et al., 2014; MATERA et al., 2009, TUDURY; POTIER, 2008), pouca atenção tem sido direcionada para o Curso de Ciências Biológicas e para o uso de animais nas aulas de zoologia, tidas até então como insubstituíveis (FISCHER; FURLAN, 2018). Diante desse argumento, o presente estudo questionou como o princípio dos $3 \mathrm{R}$ está sendo aplicado no uso de animais como recursos didáticos mundialmente e como as aulas de zoologia se inserem nesse contexto.

Apoiada em um relevante potencial de inovação foram testadas as hipóteses:

a) embora seja constatada maior intolerância ao uso de animais para atividades didáticas quando comparada com pesquisas biomédicas, acredita-se que a produção de MA para o ensino seja menos relevante que no cenário científico e que sua abrangência seja restrita às disciplinas que usam procedimentos invasivos e potencialmente geradores de conflitos; 
b) Em decorrência da atuação das CEUAs no cenário internacional somarem décadas a mais do que no nacional e da maior acessibilidade à tecnologia, espera-se encontrar diferenças na abordagem da temática MA no ensino;

c) Diante da evidência de que está aumentando o número de estudantes intolerantes ao uso de animais em aulas e de professores receptivos às novas práticas pedagógicas, acredita-se que muitos docentes estejam experimentando métodos alternativos, porém, ainda não inseridos no cenário científico, o que não lhes exime de divulgarem suas experiências na mídia popular;

d) Diante do fato do professor não ter a disposição todos os grupos zoológicos necessários para uma boa formação em sistemática da fauna, acredita-se que esteja usando métodos alternativos em suas aulas, com sucesso, mesmo sem ter consciência plena dessa conduta.

Logo, objetivou-se mapear a abordagem da temática "métodos alternativos em animais" em textos científicos e populares com veiculação de conteúdo atrelada à aplicação do princípio dos 3R no uso de métodos alternativos, acrescida da análise de planos de aula de zoologia. Os dados do presente estudo foram analisados sob a perspectiva da Bioética, visando contribuir para uma formação ética e moral de biólogos e professores de ciências de biologia, bem como a reflexão dos educadores para a necessidade de se ministrar suas aulas de forma mais humanitária e ética.

\section{MÉTODOS}

\section{Mapeamento do conteúdo científico sobre métodos alternativos ao uso animal nas atividades acadêmicas}

O conteúdo científico foi recuperado através da ferramenta Google Acadêmico, sendo a pesquisa realizada com o navegador desprovido de login, a fim de diminuir o viés dessa ferramenta quanto ao direcionamento das respostas. Para tal, foram utilizados como descritores, em português e inglês, seis termos baseados nos princípios dos 3R, promulgado pela DBCA (CONCEA, 2016): métodos alternativos ao uso de animais; métodos substitutivos ao uso de animal; simulador para modelo animal; animal alternative methods; animal replacement methods; animal simulation models. Foi acrescida a busca no portal da CAPES, na Biblioteca Digital Brasileira de teses e dissertações (BDTD), bem como análise de material complementar obtido de fontes diversas tais como: pesquisas citadas em artigos analisados e textos obtidos de consultas paralelas. Os textos recuperados foram arquivados e categorizados segundo: a) local; b) ano de veiculação; c) se o estudo era teórico/prático; d) curso destinado à pesquisa; e) disciplina; f) objetivo do trabalho; g) problemas identificados; h) procedimentos. Para cada descritor foram analisados os 100 primeiros registros.

\section{Mapeamento do conteúdo popular sobre métodos alternativos ao uso animal em atividades acadêmicas}

$\mathrm{Na}$ segunda etapa do estudo, foi categorizado o conteúdo popular disponível em sites, blogs e redes sociais presentes no meio digital sobre MA. Foi utilizado como motor de busca o Google.com e o Facebook, desprovidos de login. Para tal, foram utilizados como descritores, em português e inglês, os mesmos termos da avaliação científica. As páginas recuperadas foram arquivadas e categorizadas conforme: a) data; b) instituição; c) ferramenta (blog, site, notícias, portal, redes sociais); d) objetivos; f) 
problemas identificados; g) número de inscritos, visualizações, curtidas e compartilhamento; h) comentários. Para cada descritor foram analisados os 100 primeiros registros.

Em ambas as abordagens, a homogeneidade dos parâmetros (conteúdo; recursos; animais; tipos de aula; objetivo; métodos; resultados; problemas e conclusão), conforme as variáveis (nacional e internacional), foi acessada por meio do teste não paramétrico do qui-quadrado, tendo como hipótese nula a homogeneidade nas proporções das categorias obtidas, com uma confiança de $95 \%$ e erro de 5\%.

\section{Análise de planos de aulas de Zoologia}

O mapeamento dos procedimentos didáticos relacionados com aulas de Zoologia e a verificação do uso de métodos alternativos se deu por meio da análise de planos de aula disponíveis no meio digital. Para tal, foram recuperados os planos de aula, utilizando a ferramenta Google.com desprovida de login e com os descritores: plano de aula (adicionado o nome do filo animal). Isso foi feito com base na classificação proposta por Brusca e Brusca (2018), a saber: Protozoa, Porifera, Placozoa, Cnidaria, Ctenophora, Platyhelmintes, Mesozoa, Nemertea, Gnathostomulida, Gastrotricha, Kynorincha, Locifera, Nematoda, Nemathomorpha, Rotifera, Acanthocephala, Entoprocta, Annelida, Mollusca, Pogonophora, Spuncula, Echiura, Arthropoda: Chelicerata, Myriapoda, Insecta, Crustacea, Onychophora, Phorona, Brachiopoda, Echonodermata, Chaetognatha, Hemichordata, Cephalochordata, Tunicata, Vertebrata: Peixes, Anfíbios, Répteis, Aves, Mamíferos. Foram recuperados os 10 primeiros planos de cada categoria. O conteúdo foi categorizado conforme: a) turma destinada a atividade; b) tema da aula; c) objetivos gerais e específicos; d) conteúdos; e) metodologias; f) avaliações empregadas e agrupadas segundo as variáveis Invertebrados e Vertebrados.

A homogeneidade dos parâmetros (recursos, tipo de aula, avaliação, modelo animal) conforme as variáveis (invertebrados e vertebrados) foi acessada por meio do teste não paramétrico do qui-quadrado, tendo como hipótese nula a homogeneidade nas proporções das categorias obtidas, com uma confiança de $95 \%$ e erro de $5 \%$.

\section{RESULTADOS}

\section{Mapeamento do conteúdo científico sobre métodos alternativos ao uso animal nas atividades acadêmicas}

A amostra dos dados do presente levantamento, relativa a 600 textos científicos, atestou maior proporção de viés para o ensino nos conteúdos nacionais e predomínio da pesquisa nos textos estrangeiros (Tabela 1). Dos textos nacionais, a ampla maioria foi oriunda da região Sudeste (52,4\%) e Sul $(26,5 \%)\left(\chi_{(4)}^{2}=204 ; \mathrm{P}<0,01\right)$, quando comparados com Nordeste $(13 \%)$, Centro-oeste $(6,1 \%)$ e Norte $(2 \%)$. Já para os internacionais, a maior parte foi originário da América do Norte $(49,4 \%)$ e Europa $(38,1 \%)\left(\chi_{(4)}^{2}=330 ; \mathrm{P}<0,01\right)$, quando comparados com o continente australiano $(9,2 \%)$, asiático $(5,1)$ e africano $(3,1 \%)$. A maioria significativa dos textos nacionais recuperados referia-se a aulas de zoologia ministrada para área de biológicas $\left(\chi_{(2)}^{2}=22 ; \mathrm{P}<0,01\right)$, enquanto nos textos estrangeiros eram das áreas biomédicas $\left(\chi_{(2)}^{2}=46,5 ; \mathrm{P}<0,01\right)$ (Tabela 1$)$. Entre os recursos predominaram os modelos e softwares tanto no âmbito nacional $\left(\chi_{(4)}^{2}=32,1 ; \mathrm{P}<0,01\right)$ quanto internacional $\left(\chi_{(4)}^{2}=229,8 ; \mathrm{P}<0,01\right)$. 
Tabela 1. Frequência relativa da categorização do mapeamento do conteúdo científico sobre Métodos Alternativos ao uso de Animais.

\begin{tabular}{|c|c|c|c|}
\hline & & $\begin{array}{r}\text { Nacional } \\
(\mathrm{N}=300)\end{array}$ & $\begin{array}{c}\text { Internacional } \\
\qquad(\mathbf{N}=300)\end{array}$ \\
\hline \multirow{7}{*}{$\stackrel{\circ}{\rightleftarrows}$} & Ensino & $57,4 \%(*)$ & $18,4 \%$ \\
\hline & Pesquisa & $42,6 \%$ & $81,6 \%\left(^{*}\right)$ \\
\hline & Prática & $87,4 \%(*)$ & $38,3 \%$ \\
\hline & Teórica & $12,6 \%$ & $61,7 \%\left(^{*}\right)$ \\
\hline & Levantamento Bibliográfico & $53 \%(*)$ & $44.4 \%(*)$ \\
\hline & Prático & $38 \%$ & $54,8 \%(*)$ \\
\hline & Questionário & $9 \%$ & $0,8 \%$ \\
\hline \multirow{4}{*}{ ڤ્⿱ } & Biológicas & $39,4 \%(*)$ & $19,7 \%$ \\
\hline & Medicina e Enfermagem & $16 \%$ & $51,3 \%\left(^{*}\right)$ \\
\hline & Agrárias & $24,6 \%$ & $29 \%$ \\
\hline & Ensino Básico & $20 \%$ & $0 \%$ \\
\hline \multirow{7}{*}{ 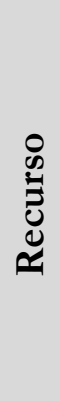 } & Não especificado & $68,4 \%$ & $65,2 \%$ \\
\hline & Especificados: & $31,6 \%$ & $34,8 \%$ \\
\hline & $3 R ` s$ & $2,2 \%$ & - \\
\hline & Áudio Visual & $7,4 \%$ & - \\
\hline & Cadáver quimicamente conservado & $13,8 \%$ & $1 \%$ \\
\hline & Modelo & $50 \%(*)$ & $64,4 \%\left(^{*}\right)$ \\
\hline & Software & $26,6 \%$ & $34,6 \%$ \\
\hline
\end{tabular}

A homogeneidade da amostra das categorizações foi aferida por meio do teste do qui-quadrado, sendo os valores significativamente maiores acompanhados de asterisco $(*)$

Fonte: dados da pesquisa

A pesquisa resultou na inclusão de 32 textos da amostra integrativa acrescida de seis artigos da amostra complementar. Entre os cursos amostrados predominou Medicina Veterinária $(78,1 \%)\left(\chi_{(3)}^{2}=\right.$ 68,2; P <0,01) quando comparado com Medicina (12,5\%), Psicologia (3,1\%) e Farmácia (3,1\%). Entre os animais, a maioria significativa substituiu o uso do cão $(45,4 \%)\left(\chi_{(3)}^{2}=17,7 ; \mathrm{P}<0,01\right)$, quando comparado com ratos $(18,1 \%)$, felinos (9\%), suínos (3\%) e não especificados (3\%). Dentre as disciplinas destacou-se Técnica Cirúrgica $(45,4 \%) \quad\left(\chi_{(3)}^{2}=12,5 ; \mathrm{P}<0,01\right)$, quando comparada com Anatomia e Fisiologia $(18,1 \%)$, Análise de Comportamento (3\%), Anestesiologia (6\%), Farmacologia (3\%), Histologia (6\%), Inseminação Artificial (3\%), Laboratório de Análises Práticas (3\%), Ortopedia (3\%) e Punção Venosa $(6 \%)$. Entre os recursos utilizados, a maioria significativa indicou o modelo $(25 \%)\left(\chi_{(6)}^{2}=12 ; \mathrm{P}<0,01\right)$, quando comparado com cadáver (21,8\%), manequim $(15,6)$, software $(15,6 \%)$, audiovisual $(9,3 \%)$, artesanais $(6,25 \%)$ e lâminas $(3,1 \%)$. 
A análise dos textos específicos evidenciou que $63 \%$ foram relativos a relatos de experiências, tanto de propostas de modelos para substituição de animais vivos quanto de métodos de preservação de cadáveres e de avaliação do aprendizado do estudante, primando pela eficiência pedagógica. Nos artigos analisados: foi evidenciado como benefícios: a) o cuidado com o uso da técnica a fim de preservar os espécimes; b) supressão de técnicas já repetidas e ineficientes; c) reflexões éticas do uso de animais; d) possibilidades para a substituição do animal por modelos que sejam economicamente viáveis e que possibilitem condutas éticas na relação dos animais utilizados para aulas. Dos artigos, apenas quatro se referiam a aulas de Zoologia, sendo três relativos à levantamento bibliográfico e um analisando a percepção de acadêmicos sobre aulas práticas com animais em Zoologia. Os artigos teóricos abordaram textos que elucidavam métodos alternativos, considerando o histórico em cenários nacionais e internacionais e análises de pesquisas já realizadas. $\mathrm{O}$ trabalho que retratou a percepção dos acadêmicos sobre a utilização de métodos alternativos, aplicou questionários para avaliar o que pensam sobre a utilização da metodologia. Como resultado, averiguou-se que os acadêmicos gostariam de ter mais ações que substituíssem os animais em atividades práticas, visando o BEA (Figura 1). 
Figura 1. Síntese da análise qualitativa do material recuperado no meio científico e popular sobre métodos alternativos ao uso de animais no ensino.

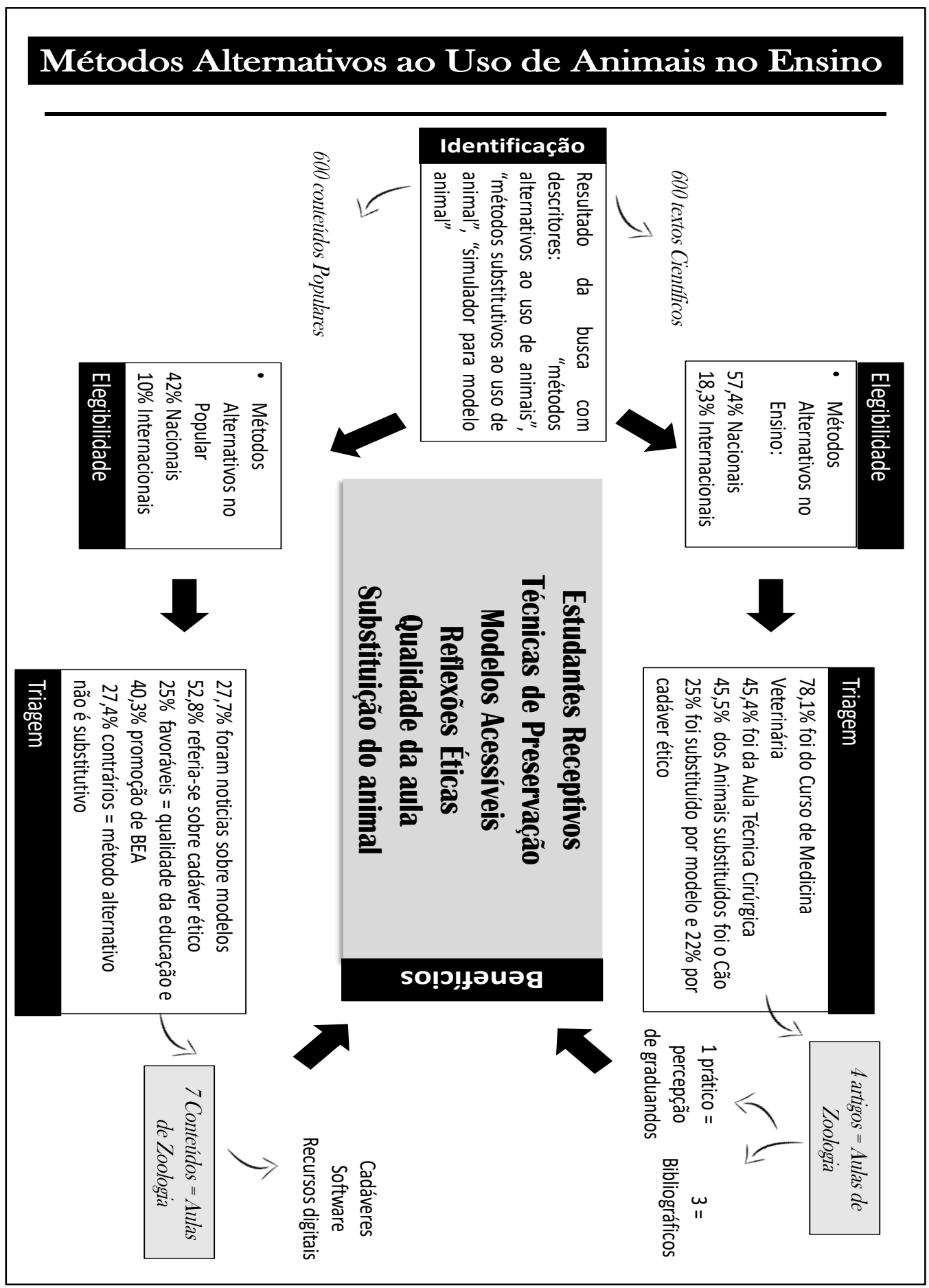

Fonte: os autores

Mapeamento do conteúdo popular sobre métodos alternativos ao uso animal em atividades acadêmicas

A amostra dos dados do presente levantamento, relativa a 600 conteúdos populares, indicou que no cenário nacional o conteúdo esteve vinculado a sites de informações, enquanto nas estrangeiras a maioria significativa esteve voltada a conteúdo científico (Tabela 2). O modo como se abordou o 


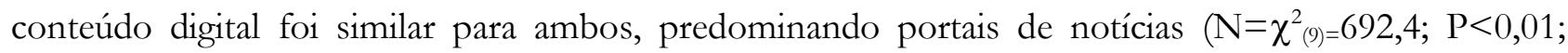
$\left.\mathrm{I}=\chi_{(9)}^{2}=899 ; \mathrm{P}<0,01\right)$. Nas páginas e vídeos analisados, quanto aos recursos utilizados para a substituição pelo modelo animal, evidenciou-se predomínio do uso de cadáveres éticos. Entre os argumentos favoráveis, destacou-se a qualidade na educação e o cumprimento do bem-estar animal. Já como contrários, predominou a impossibilidade de o método alternativo ser substitutivo $\left(\chi_{(4)}^{2}=14,3 ; \mathrm{P}<0,01\right)$.

A pesquisa integrativa resultou na inclusão de sete páginas da amostra, nas quais, embora não tenha sido especificado o curso, todos foram referentes às aulas de Zoologia. Entre os animais, destacouse o uso de cadáveres na rede social, seguido por cães, coelho e rato. Dos recursos utilizados como métodos alternativos, a maioria significativa apontou o uso de software, seguido por modelos e recursos digitais. Ao indicarem os pontos positivos para o uso de métodos alternativos, a qualidade na educação teve ênfase no recorte da pesquisa, assim como a possibilidade de substituição do animal. O ponto negativo apontado na amostra indicou a falta de homogeneidade entre os professores ao utilizar o métodos alternativos, afirmando que há restrição na qualidade do ensino, que apenas com a presença de animais seria possível promover o aprendizado de forma eficiente (Figura 1). 
Tabela 2. Frequência relativa do Mapeamento do conteúdo popular sobre uso de Métodos Alternativos ao uso de Animais.

\begin{tabular}{|c|c|c|c|c|c|}
\hline & & \multicolumn{2}{|c|}{ Sites e Blogs } & \multicolumn{2}{|c|}{ Redes Sociais } \\
\hline & & Nacional & Internacional & Nacional & Internacional \\
\hline \multirow{10}{*}{ 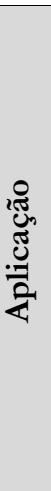 } & Científico & $34,8 \%$ & $48,8 \%(*)$ & $21 \%(*)$ & $38 \%(*)$ \\
\hline & Site & $49,3 \%(*)$ & $43,5 \%$ & $1 \%$ & $5,8 \%$ \\
\hline & Guia de criação animal & $0,3 \%$ & - & $1 \%$ & $1,8 \%$ \\
\hline & Divulgação de Evento & $2,5 \%$ & - & $11 \%$ & $15 \%$ \\
\hline & Noticias & $6,3 \%$ & $4 \%$ & $58 \%(*)$ & $34 \%(*)$ \\
\hline & Normativas & $1,7 \%$ & $1 \%$ & $5 \%$ & $1,8 \%$ \\
\hline & Youtube & $0,3 \%$ & - & - & - \\
\hline & $B \log$ & $3,9 \%(*)$ & - & $2 \%$ & $1,8 \%$ \\
\hline & Compra e venda & $0,9 \%$ & $0,9 \%$ & $1 \%$ & - \\
\hline & Doação & - & $1,8 \%$ & - & $1,8 \%$ \\
\hline \multirow{3}{*}{ 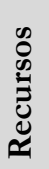 } & Software & $26,8 \%(*)$ & $30 \%$ & $10,8 \%$ & $21,3 \%$ \\
\hline & Modelo & $25,2 \%(*)$ & $43,3 \%(*)$ & $70,2 \%(*)$ & $53,7 \%(*)$ \\
\hline & Recursos Digitais & $19,4 \%$ & $13,3 \%$ & $19 \%$ & $25 \%$ \\
\hline \multirow{8}{*}{$\begin{array}{l}\frac{0}{0} \\
\frac{0}{0} \\
\sum\end{array}$} & Membrana animal & $5,2 \%$ & - & $11,3 \%$ & $3,5 \%$ \\
\hline & Animais de laboratório & $10,5 \%$ & $15,7 \%$ & $22,5 \%(*)$ & $9 \%$ \\
\hline & Cadáveres & $52,6 \%$ & $36,8 \%(*)$ & $38,6 \%(*)$ & $34 \%(*)$ \\
\hline & Cachorro & $10,5 \%$ & - & $6,8 \%$ & $3,5 \%$ \\
\hline & Peixes & $10,5 \%$ & - & $4,5 \%$ & $1,7 \%$ \\
\hline & Porco & $5,2 \%$ & - & $4,5 \%$ & - \\
\hline & Rã & $5,2 \%$ & - & $6,8 \%$ & - \\
\hline & Humanos & - & $47,5 \%$ & $6,8 \%$ & $48,3 \%(*)$ \\
\hline \multirow{4}{*}{ 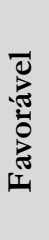 } & Qualidade na Educação & $40,4 \%(*)$ & $10 \%$ & $44,2 \%(*)$ & $31 \%(*)$ \\
\hline & $\begin{array}{l}\text { Cumprindo o bem-estar } \\
\text { animal }\end{array}$ & $27,4 \%$ & $43,4 \%(*)$ & $31,2 \%(*)$ & $57 \%(*)$ \\
\hline & Economia & $16,1 \%$ & $13,3 \%$ & $14,8 \%$ & $7 \%$ \\
\hline & Avanço tecnológico & $16,1 \%$ & $33,3 \%$ & $9,8 \%$ & $5 \%$ \\
\hline \multirow{6}{*}{ 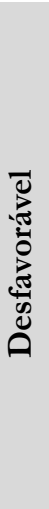 } & Investimento em pesquisas & $9,5 \%$ & $20 \%$ & $20,5 \%$ & $18,5 \%$ \\
\hline & $\begin{array}{l}\text { Fatores econômicos } \\
\text { inviabilizam }\end{array}$ & $18,8 \%$ & $16,6 \%$ & $11,8 \%$ & $22,2 \%$ \\
\hline & $\begin{array}{l}\text { Fatores econômicos } \\
\text { inviabilizam }\end{array}$ & $18,8 \%$ & $16,6 \%$ & $11,8 \%$ & $22,2 \%$ \\
\hline & $\begin{array}{l}\text { MA não pode ser } \\
\text { substitutivo }\end{array}$ & $45,3 \%(*)$ & $43,4 \%(*)$ & $44,2 \%(*)$ & $44,5 \%(*)$ \\
\hline & $\begin{array}{l}\text { Falta unanimidade entre } \\
\text { pesquisadores }\end{array}$ & $13,2 \%$ & $20 \%$ & $23,5 \%$ & $14,8 \%$ \\
\hline & $\begin{array}{l}\text { Falta adesão pelos } \\
\text { professores }\end{array}$ & $13,2 \%$ & - & & \\
\hline
\end{tabular}

*A homogeneidade da amostra das categorizações foi aferida por meio do teste do qui-quadrado, sendo os valores significativamente maiores acompanhados de asterisco $(*)$

Fonte: dados da pesquisa 


\section{Análise de planos de aulas de Zoologia}

A análise dos planos de ensino evidenciou predomínio de aulas sobre invertebrados (85,8\%) quando comparado a vertebrados $(14,2 \%)\left(\chi_{(1)}^{2}=147,3 ; \mathrm{P}<0,01\right)$, bem como aulas expositivas $\left(\chi_{(1)}^{2}=2,8\right.$; $\mathrm{P}<0,01)$ e em Instituições de Ensino Superior (IES) Federais (59,5\%), quando comparadas com as Privadas (20,5 \%), Estaduais $(18,8 \%)$ e Municipais $(1,2 \%) \quad\left(\chi_{(3)}^{2}=225,5 ; \mathrm{P}<0,01\right)$. As regiões de prevalência foram a Sul $(41 \%)$ e Sudeste $(33 \%)$, quando comparadas com Nordeste $(9,6 \%)$, Norte $(8,4 \%)$ e Centro-Oeste $(8 \%)\left(\chi_{(4)}^{2}=128,1 ; \mathrm{P}<0,01\right)$. A maioria significativa dos planos de ensino da amostra foram dos cursos das áreas biológicas (75\%), seguidos pelos do ensino básico e técnico (14,4\%), os de agrárias $(6,1 \%)$ e os de biomédicas $(4,5 \%)\left(\chi_{(3)}^{2}=330 ; \mathrm{P}<0,01\right)$ (Tabela 3). Entre os recursos, prevaleceu a utilização de animais fixados $(77,7 \%)\left(\chi_{(6)}^{2}=28,6 ; \mathrm{P}<0,01\right)$.

Tabela 3. Frequência relativa do Mapeamento dos planos de aula sobre Métodos Alternativos em aulas de Zoologia

\begin{tabular}{|c|c|c|c|c|}
\hline & & Vertebrados & Invertebrados & Total \\
\hline & Especificados: & & & \\
\hline & Prova & $54,5 \%(*)$ & $46,6 \%(*)$ & $47,7 \%\left(^{*}\right)$ \\
\hline & Trabalho & $35 \%(*)$ & $40 \%(*)$ & $39,5 \%(*)$ \\
\hline & Participação & $10,5 \%$ & $13,4 \%$ & $12,8 \%$ \\
\hline$\therefore, \pi$ & Teórico & $55,2 \%(*)$ & $60,4 \%(*)$ & $60 \%(*)$ \\
\hline$\dot{H}=\vec{z}$ & Prático & $44,8 \%$ & $39,6 \%$ & $40 \%$ \\
\hline & Especificados & & & \\
\hline & Organismos Vivos & $0 \%$ & $17,2 \%$ & $7,7 \%$ \\
\hline : & Lâminas & $22,3 \%(*)$ & $34,4 \%$ & $16,4 \%(*)$ \\
\hline हुं & Animais Fixados & $77,7 \%(*)$ & $36,5 \%$ & $19,8 \%(*)$ \\
\hline & Animais a seco & - & $2,3 \%$ & $0,9 \%$ \\
\hline & Animais conservados & - & $1 \%$ & $0,4 \%$ \\
\hline & Método Alternativo & - & $8,6 \%$ & $3,8 \%$ \\
\hline
\end{tabular}

*A homogeneidade das categorias de cada parâmetro avaliativo foi testada por meio do teste do qui-quadrado sendo os valores significativamente maiores $(\mathrm{P}<0,05)$ acompanhados de asterisco $(*)$.

$\mathrm{Mi}=$ Microscópio $-\mathrm{V}=$ vídeos $-\mathrm{I}=$ imagens $-\mathrm{M}=$ modelos comparados $-\mathrm{MFP}-$ modelos feitos por professor ou técnico - MFA - modelos feito alunos $-\mathrm{I}=$ ilustrações $-\mathrm{L}=$ livros; $\mathrm{S}=$ software $-\mathrm{PPT}=$ power point $\mathrm{DSW}=$ Data Show $-\mathrm{Q}=$ quadro $-\mathrm{RP}=$ retro projetor $-\mathrm{Tv}-\mathrm{AC}=$ aula de campo

Fonte: dados da pesquisa

\section{DISCUSSÃO}

Os dados do presente estudo permitiram traçar o panorama da abordagem dos métodos alternativos no cenário científico e popular nacional e internacional e nos planos de aula de zoologia, atestando que a substituição dos animais como recurso didático ainda é escassa e restrita tanto em termos de distribuição geográfica quanto em segmentos que usam procedimentos mais invasivos, cuja identificação e monitoramento deve envolver a capacitação ética e técnica do professor. 
O fato de os artigos internacionais focarem nos métodos alternativos voltados para pesquisa e nos nacionais prevalecerem as atividades acadêmicas pode ser reflexo de uma maior maturidade do meio acadêmico e do setor industrial norte-americano e europeu com relação à aplicação do princípio dos 3R no uso de animais como modelos experimentais, com vias a favorecer a substituição. Tréz (2018), em sua reflexão sobre a aplicação dos $3 \mathrm{R}$, ressaltou que a maior crítica envolvida nesse segmento se refere ao enaltecimento da redução e do refinamento em prol da substituição. Justamente o viés bem-estarista legitima a utilização dos animais, mesmo condicionada à determinadas regras. De fato, é possível encontrar, no meio científico nacional, além de críticas aos 3R e à DBCA (VILLIERS; SOMMERVILLE, 2005), pesquisas que sugerem a promoção do bem-estar animal como conduta de integridade na pesquisa (FISCHER et al., 2016; FISCHER; SANTOS, 2017; BROOM; MOLENTO, 2004; REGAN, 2006; RIVERA, 2006; HÖTZEL; MACHADO-FILHO, 2004).

Os métodos alternativos para utilização industrial e científica, mesmo reconhecidos internacionalmente, devem passar por um sistema de validação nacional realizado pelo Centro Brasileiro para Validação de Métodos Alternativos (braCVAM), vinculado ao CONCEA. Após essa etapa, são publicados como resolução normativa e no diário oficial e, a partir de cinco anos, passa a ser crime usar animais para a referida prática. A própria DBCA (CONCEA, 2016) é prudente ao recomendar a utilização de métodos alternativos reconhecidos e que não comprometam os resultados, sendo sugerido, antes da validação, que os testes sejam comparados com o modelo animal. Logo, alcançar métodos alternativos para procedimentos experimentais demanda investimento, pesquisa e produção, compreensivelmente atrelados às demandas específicas do mercado. Segundo Morales (2008), nesse segmento será virtualmente impossível substituir o uso de animais, pois a produção de medicamentos demanda um substrato orgânico complexo para testar seu processo de metabolização, contando a ciência apenas com métodos alternativos e complementares, processo que tem angariado mais sucesso na área da toxicologia (BEDNARCZUK et al., 2010; CONCEA, 2016).

Os métodos alternativos no ensino já possuem uma conotação experimental e estão ao alcance dos professores, que podem usar material e técnicas alternativas que não demandem tanto investimento e necessidade de validação por órgãos oficiais. Acresce-se ao meio mais receptivo o fato de estudantes estarem mais intolerantes ao uso de animais para procedimentos didáticos do que os científicos (FISCHER; TAMIOSO, 2013; FEIJÓ et al., 2008). Mesmo assim, foi possível atestar, com o presente mapeamento, que essas pesquisas têm beneficiado, prioritariamente, a área de Medicina Veterinária e, em especial, a disciplina de Técnica Cirúrgica. Segundo Passerino et al. (2014), essas aulas têm gerado um conflito enfrentado pelas CEUAs, uma vez que instituições de ensino que comportam o curso de Medicina e Medicina Veterinária precisa gerir as decisões do porquê a primeira alega ser imprescindível para formação do médico treinar em animais, especialmente porcos, enquanto que, para os estudantes de Medicina Veterinária, usa-se cadáveres. Segundo os autores, o Conselho Federal de Medicina Veterinária condena a destituição da vida de um animal saudável para treinamento, pois, além dessa conduta atentar contra o próprio código de ética (CFMV, 2008), os estudantes terão a oportunidade de fazer residência e estágios no hospital escola. Diante dessa necessidade e toda polêmica pregressa no uso de cães semi-domiciliados para essas práticas, a área da Medicina Veterinária investiu em pesquisa e validação de métodos que englobam desde o uso de manequins e simuladores com alta agregação de tecnologia (MAGALHÃES; ORTÊNCIO-FILHO, 2006; TUDURY; PORTIER, 2008) até métodos mais artesanais (COSTA-NETO, 2011; CAZARIN et al., 2004). O grupo de membros das CEUAs, que 
discutiu meios de mitigar esse conflito avaliado por Passerino et al. (2014), concordou com o uso de animais, porém, só em estágios avançados do curso e da disciplina, sendo que o treinamento prévio deve ser realizado em material substitutivo.

O uso de modelos e softwares como alternativas ao animal evidenciado tanto no cenário nacional quanto internacional, indica a possibilidade de permitir ao estudante acessar de maneira mais eficiente estruturas e peças anatômicas, as quais nem sempre são possíveis de serem vistas no animal, principalmente por se desgastarem com a manipulação frequente. Acresce-se a isso a vantagem do acompanhamento personalizado, a tolerância a erros e a possibilidade de correção dos mesmos por meio da repetição dos processos, respeitando seu tempo de aprendizado individual (FISCHER; FURLAN, 2018). Magalhães e Ortêncio-Filho (2006) relataram que esse processo, há mais de 10 anos, é utilizado em universidades nacionais e estrangeiras com o objetivo de treinamento para simular órgãos, membros ou mesmo animais inteiros, para a realização de treinamento e simulação - de funções fisiológicas, habilidades clínicas e práticas cirúrgicas. Contudo, ainda existem divergências quanto a sua eficácia, uma vez que o tradicionalismo na educação permanece como uma vertente imponente, sendo que o uso de tecnologias causa estranheza naqueles que não o dominam. A resistência e descrédito dos estudantes foi atestada por Tudury e Portier (2008) com acadêmicos do Curso de Ciências Biológicas e por Villiers e Sommerville (2005), estudo no qual os discentes apontaram a preferência por dissecação animal. Por outro lado, outros pesquisadores como Feijó et al. (2008) têm registrado a adesão de estudantes da área da saúde à substituição animal, considerando como balizadores o sofrimento e o bem-estar animal.

Os softwares englobam análises morfológicas, fisiológicas e evolutivas. Segundo Fischer (2017), a tecnologia por meio da realidade virtual, agregando inovação, criatividade e eficiência, possibilita a visualização de processos bioquímicos e respostas fisiológicas decorrentes de aplicação de substâncias. Os simuladores possibilitam que se misturem distintas substâncias e consequente efeito em sistemas e órgãos, sem causar nenhum tipo de dor e sofrimento real. O curso de Psicologia tem, gradativamente, substituído os ratos em aula de modelagem comportamental por software como o Sniff, que permite ao estudante repetir todos os processos - e levar seu animal no pendrive sem envolver o estresse da manipulação e o sofrimento da privação de água ou alimento (FISCHER, 2017).

O uso de cadáveres quimicamente conservados, como atestado no presente mapeamento, constitui uma técnica utilizada em IES, como forma de substituir as obtenções frequentes de animais. Segundo Jukes e Chiuia (2003), a técnica consiste em plastificar animais dissecados e preservados, cujos tecidos moles são substituídos quimicamente por plástico (MAGALHÃES; ORTÊNCIO-FILHO, 2006), fornecendo ao discentes uma experiência tátil e espacial. Esse procedimento é bem aceito em universidades como a de Ohio e na Universidade de Zurique, na qual o curso de Medicina Veterinária utiliza modelos confeccionados a partir de poliuretano inodoro, que viabiliza ao educando práticas de habilidades de técnicas cirúrgicas sem o custo de realizar eutanásia nos animais (AUER, 1994). Contudo, a DBCA (CONCEA, 2016) alerta para o uso de cadáver ético, ou seja, que esses animais tenham origem idônea e que a IES, conjuntamente com a CEUA, monitore a doação e as condições de morte desses animais. Esses procedimentos se fizeram necessário diante da prática de eutanásia em animais sadios minutos antes da aula, apenas para constar que não estavam vivos (PASSERINO et al., 2014). O principal argumento contra o uso de cadáveres para técnicas cirúrgicas é a ausência de sangue e a consequente impossibilidade de o estudante treinar situações que demandem respostas rápidas e, principalmente, controle emocional (PASSERINO et al., 2014). Para suprir essa demanda, Matera et al. (2009) 
desenvolveram a técnica do cadáver que sangra, em que, através do mesmo grande vaso por onde é retirado o excesso de sangue, é injetada a solução em todo o sistema circulatório, o que faz com que a cobaia não se deteriore e ainda possa ser congelada e descongelada de oito a dez vezes (MATERA et al., 2009).

A presente análise evidenciou que, em certas atividades acadêmicas como a punção venosa e aferição de pressão arterial, ao invés de se utilizarem animais, são os próprios acadêmicos os modelos para seus colegas no decorrer das aulas (MAGALHÃES; ORTÊNCIO-FILHO, 2016. Vieira (2006) alertou que, igualmente, existem diretrizes éticas internacionais que normatizam o uso de seres humanos nesse contexto, redigidas pelo Conselho para Organização Internacional de Ciências Médicas (CIOMS), em colaboração com a Organização Mundial de Saúde (OMS), que reconheceram como legal desde que haja aprovação do protocolo através de uma comissão de revisão ética. Neste recorte de pesquisa, notouse a existência das experiências envolvendo seres humanos, o que fomenta a necessidade de regulamentação da prática, de forma a se cumprir eticamente os protocolos, a fim de que não traga prejuízo para os participantes.

Embora o meio científico não esteja contribuindo satisfatoriamente para a disseminação de experiências com métodos alternativos ao uso de animais na educação, a consulta a conteúdos populares evidenciou que corresponde a quase metade das referências recuperadas no cenário nacional. Muitos professores têm tomado a incitava de procurar métodos alternativos, seja por não quererem se submeter às burocracias demandadas pela CEUA (FISCHER et al., 2014), ou pelas existência de estudantes que têm se mostrados mais sensíveis ao uso de animais em aulas (FISCHER; TAMIOSO, 2013; FEIJÓ et al., 2008). A internet, para Valerio (2005), marca o advento de novos meios de se obter informações, tanto na perspectiva popular quanto na acadêmica. Ressalta-se que a transposição das experiências para estudos bem estruturados, em que se avalie criteriosamente a eficiência do método, se constitui como forma de validá-lo como substitutivo. Logo, é fundamental que essas experiências sejam divulgadas e que haja uma mobilização dos docentes em desenvolver estudos estruturados e que revertam, além do benefício imediato para o estudante, em um conjunto de dados para aprimorar as técnicas nesse segmento.

A análise qualitativa dos conteúdos por meio do recorte proporcionado pela presente pesquisa aponta que substituir o uso de animais revela uma preocupação educacional, valendo-se de metodologias humanitárias na experimentação animal (FISCHER et al., 2014). Os resultados enaltecem o maior comprometimento com a qualidade de educação através do métodos alternativos, acrescido da promoção do bem-estar animal, corroborando autores como Jukes e Chiuia (2003) e Greif (2003). Greif (2003) defendeu como vantagens na utilização de métodos alternativos: a baixos custos, quando comparados à manutenção de biotérios e preparação de animais; vida útil indeterminada; melhores aprendizados ao utilizar software e modelos artificiais; respeito à evolução cognitiva do acadêmico, possibilitando a repetição quantas vezes for necessário; aprendizado balizado por princípios éticos.

O principal argumento contra a utilização de métodos alternativos no recorte desta pesquisa foi que eles não podem ser substitutivos pelo uso de animais. Tal afirmação é polêmica, movimentando discussões acaloradas (LEVAI, 2010). O uso da técnica tradicional e o embasamento empírico de que não existe a possibilidade de educação com métodos substitutivos vão contra a visão de Costa-Neto (2011), que afirmou que é questionável o melhor rendimento com animais em aulas práticas. Há, ainda, a questão de as aulas práticas com a manipulação animal ferirem os princípios do bem-estar animal, prejudicando igualmente a formação do estudante. Rodrigues (2010) reafirma que as disciplinas que 
dependem de animais, por si sós, não conferem a habilidade de bons profissionais, sugerindo que o aluno deverá se capacitar em atividades complementares, como estágios e iniciação científica. Diniz et al. (2006) complementaram que a função do educador é ensinar técnicas, mas, também, induzir valores e posturas éticas nos alunos, promovendo um conhecimento balizado em ações humanitárias, vislumbrando os agentes morais envolvidos para que transcorra o aprendizado sem prejuízos. Fischer (2017) listou as condutas esperadas de docentes, discentes e colaboradores no uso de animais, alertando que o estudante deve ser instruído sobre métodos apropriados de manejo, que seja competente para realizar as tarefas com cuidado, que tenha responsabilidades éticas e legais e conhecimento de todo processo e procedimentos relacionados com a aplicação do princípio do 3R. A capacitação e certificação dessas competências no estudante é uma determinação legal (CONCEA, 2016) que promove a autorregularão desses processos no meio acadêmico.

Nos resultados obtidos com o levantamento, não foi evidenciada nenhuma menção à cláusula da objeção de consciência, a qual se constitui de um instrumento legal importante para o estudante que se sinta constrangido em presenciar uma aula com animais (LEVAI, 2010). A exigência que o docente deve preparar aulas com métodos alternativos para esses estudantes tem amparo na Legislação da DCBA (CONCEA, 2016) e na Lei Estadual17.442/2012. Essa medida vem como forma de atenuar um processo comum em cursos que usam animais na sua essência, como Ciências Biológicas, Medicina Veterinária e Zootécnica, em que os estudantes que ingressam no curso se deparam com conflitos morais, mas, a postura do professor e alegação da necessidade do processo para formação profissional e importância da experimentação para o progresso da Ciência, cria uma crescente dessensibilização (REIS; TRÉZ, 2009; TRÉZ, 2011; FISCHER, 2017). Esse processo de dessensibilização com relação ao sofrimento animal vem sendo promovida desde a Revolução Científica maximizada por interesses econômicos, industriais e científicos (VARGAS; CERVI, 2012). Reis e Tréz (2009) acreditam que os estudantes diminuem seus incômodos com a experimentação animal ao longo do curso, alterando suas percepções para lidarem com o conflito. A experimentação é, então, justificada pelas recompensas pessoais e sociais e exposição a situações opressoras, cuja idoneidade dos processos acadêmicos se colocam como soberanos, conduzindo o estudante a escolher pelo silêncio e respeito à prática (TRÉZ, 2011).

A análise dos planos de aula confirmou a hipótese de que o professor está usando métodos alternativos com sucesso, mesmo sem ter consciência plena dessa conduta. A pesquisa evidenciou prevalência para a substituição de animais invertebrados frente aos vertebrados. Contudo, essa conduta é resultado de uma impossibilidade técnica e não ética, uma vez que é decorrente de uma maior dificuldade em obter, conservar e até mesmo dissecar animais invertebrados (FISCHER; FURLAN, 2018), obrigando, assim, o professor a buscar alternativas bibliográficas, digitais, modelos e intervenções que apresentem para o estudante o animal e suas características. Fato esse que se contrapõe ao discurso da inviabilidade da extinção do uso de animais para essas aulas (FISCHER; FURLAN, 2018). Embora essa evidência seja relevante para apoiar a viabilidade da inserção dos métodos alternativos no ensino da Zoologia, o descomprometimento ético é uma questão preocupante que não deve ser negligenciada. A proteção de vertebrados encontra embasamento legal, apoiado na argumentação da impossibilidade de atestar a senciência nos invertebrados (ELWOOD, 2011; CROOK; WALTERS, 2011). Fischer e Santos (2017) elucidaram que a diferenciação entre invertebrados e vertebrados é unicamente acadêmica, tendo em vista que $95 \%$ da fauna é composta por invertebrados, os quais desempenham papéis fundamentais 
como decompositores, polinizadores e participantes importantes na cadeia alimentar, sendo também controladores de populações. A pouca lucidez de informações sobre os invertebrados, interpelada por Fischer et al. (2016), a respeito de sua senciência, aliada à atribuição cultural da maleficência, recebe aprovação por parte da maioria da sociedade, inclusive os acadêmicos e pesquisadores, a não lhes conferir o atributo de status moral, considerando-os incogniscientes, inconscientes e não sencientes. Consequentemente, essa conduta potencializa a vulnerabilidade tanto dos animais quanto dos estudantes, que têm a construção da sua autonomia crítica comprometida em decorrência do conflito conceitual/ético do porquê deve-se ser ético com alguns animais (vertebrados), mas não com outros (invertebrados). Isso entra em conflito inclusive com o código de ética do Biólogo, que promulga que a vida, em todas as suas manifestações, deve ser preservada e respeitada (CFBio, 2002).

A principal forma de avaliação associada com métodos tradicionais, como provas e trabalhos, remete ao uso de tais recursos educacionais conservadores (DEMO, 2002), os quais contribuem para reforçar a legitimidade do uso de animais. Segundo Demo (2002), o processo tradicional de avaliação da aprendizagem desconsidera importantes aspectos da progressão estudantil e, assim como a implementação dos métodos alternativos ao uso de animais como recursos didáticos, demanda a implementação de pedagogias inovadoras como o ensino por competências (ABRANTES, 2001) e o uso de metodologia ativas (MITRE et al., 2008). O uso coadjuvante de métodos alternativos e metodologias ativas, como forma de promover um debate coeso a respeito da utilização animal, irá formar e capacitar profissionais autônomos, alcançando dimensões afetivas e intelectuais de forma duradoura (FISCHER; FURLAN, 2018). Para Almeida e Valente (2012), o tradicionalismo educacional fez sentido na época em que a busca de informações era limitada, entretanto, com o advento da internet, os recursos educacionais e técnicas tradicionais podem ser substituídas. A tecnologia se constitui da integração espaço/tempo, na qual, segundo Moran (2015), o ato de ensinar e aprender estão interligados e não reclusos mais a espaços isolados, assim como sua dinamicidade exige atuação em múltiplos espaços do cotidiano e no papel do educador no equilíbrio de interagir tecnologias móveis com o aprendizado. Essa concepção corrobora o recorte da pesquisa, apontando a necessidade de renovação nos meios avaliados. Os ajustes disruptivos, para Moran (2015), são necessários para que o aluno se torne menos passional e mais ativo, tornando o professor um orientador e não um transmissor de conteúdo. Segundo Fischer e Furlan (2018), é possível associar metodologias ativas e aplicação do princípio dos 3R nas aulas de zoologia de invertebrados, principalmente vinculados ao uso eficaz de recursos didáticos envolvidos nos meios digitais.

A alegação dos docentes para uso de animais, em especial na disciplina de Zoologia, da necessidade da presença do animal real para a formação profissional, se contrapõe à realidade de que nem todos os grupos estão à disposição do docente, o que o leva a buscar métodos alternativos, mesmo de forma inconsciente. A substituição de aulas com animais se constitui de estratégia cujo questionamento está relacionado à forma que os seres humanos têm se relacionado com os animais. Para o autor, os docentes se sentiam mais confortáveis em não haver a necessidade do sacrifício animal. Esse fato corrobora com a pesquisa - que docentes, de forma não consciente, se valem de meios para não utilizar animais, usando técnicas como lâminas e animais fixados. Fischer e Furlan (2018) pontuaram, ainda, que, por mais simples e distante do modelo real que seja um métodos alternativos, cumpre um papel educativo importante, ao instigar a reflexão crítica sobre a conduta do meio acadêmico, contribuindo para formação de um profissional que questiona sua própria conduta diante de dilemas éticos. 


\section{CONSIDERAÇÕES FINAIS}

A presente pesquisa permitiu alcançar o objetivo proposto de caracterizar a substituição animal nas aulas de Zoologia, constituinte da formação do professor de ciências de biologia, mesmo diante da incipiência de abordagens da temática no recorte do estudo, e da maioria das pesquisas consistirem em estudos teóricos e pouco vinculados aos animais.

Embora os professores aleguem a necessidade inquestionável do uso de animais para o ensino da zoologia e a impossibilidade de substituição, realizam a substituição quando não tem os animais a sua disposição. A análise dos planos de aulas atestou que a substituição é corriqueira, principalmente, para invertebrados, cuja ampla diversidade e dificuldade de manutenção inviabiliza a disponibilidade de todos os grados.

O mapeamento de como os métodos alternativos têm sido abordado em diferentes meios de comunicação, para diferentes públicos, torna evidente que o caminho a ser seguido é trabalhoso, pois, timidamente, existem pesquisas que apontam a efetividade de substituir o modelo animal. Por outro lado, o pensamento tecnicista e utilitário de professores, alunos e da sociedade dificulta transpassar essa realidade e a demanda pela abolição completa dos animais como recursos didáticos. A existência de conflitos não deve ser resolvida com imposição e autoritarismo, mas por meio de bom-senso, diálogo e incentivo às soluções factíveis e que não comprometam a qualidade do profissional em formação. Considerando a existência dos colegiados das CEUA, com uma essência bioética preparada para gestão de crises em prol da proteção e cuidado do vulnerável, esses espaços deveriam intensificar a seu papel educativo. Dessa forma, estariam ensinando e tornando a substituição animal um assunto a ser mais debatido, bem como incentivando novas pesquisas, equalizando os valores inerentes à vida e ao não sofrimento, em prol da formação de cidadãos autônomos, críticos e protagonistas na manutenção da vida e da sustentabilidade do planeta. Dessa maneira, com a Bioética cumprindo o papel de interlocutora dos vulneráveis, será possível um ensino humanitário, que vise o aprendizado de forma ética e igualitária.

\section{AGRADECIMENTOS}

À Fundação Araucária pela Bolsa de Estudo

\section{REFERÊNCIAS}

ABRANTES, P. et al. Currículo Nacional do Ensino Básico. Competências Essenciais. Lisboa: Ministério da Educação, Departamento de Educação Básica, 2001.

ALMEIDA, M.E.B.; VLENTE, J.A. Integração currículo e tecnologias e a produção de narrativas digitais. Currículo sem fronteiras, v. 12, n. 3, p. 57-82, 2012.

AUER, J.A. Veterinär-chirurgischeausbildung am simulator. Alternativenzu Tierexperimenten, v. 11, n. 1, p. 44-46, 1994. 
BASTOS, J.C.F. et al. Implicações éticas do uso de animais no processo de ensino-aprendizagem nas faculdades de medicina do Rio de Janeiro e Niterói. Revista Brasileira de Educação Médica, v. 26, n. 3, p.162-170, 2002.

BASSALOBRE, J.N. Ética, responsabilidade social e formação de educadores. Educação em Revista, v. 29, n. 1, p. 311-317, 2013.

BEDNARCZUK, V.O., VERDAM, M.C., MIGUEL, M.D., MIGUEL, O.G. Testes in vitro e in vivo utilizados na triagem toxicológica de produtos naturais. Visão Acadêmica, v.11, n.2, p. 43-50, 2010.

BRASIL. Lei 9.605 De Crimes Ambientais, de 1998. Disponível em:

<http://www.planalto.gov.br/ccivil_03/leis/19605.htm>. Acesso em 29 de out. 2018.

BRASIL. Lei 11794 de 08 de Outubro de 2008. Procedimentos para uso cientifico dos animais.

Disponível em: <www.planalto.gov.br/ccivil_03/_ato2007-2010/2008/lei/111794.html>. Acesso em 29 de out 2018.

BROOM, D.M.; MOLENTO, C.F.M. Bem-estar animal: Conceito e Questões relacionadas revisão. Archives of veterinary Science, v. 9, n. 2, p. 1-11, 2004.

BRUSCA, R.C.; BRUSCA, G.J. Invertebrados. Madrid: McGraw-Hill, 2018.

CAZARIN, K.C.C.; CORRÊA, C.L.; ZAMBRONE, F.A.D. Redução, refinamento e substituição do uso de animais em estudos toxicológicos: uma abordagem atual. Revista Brasileira de Ciências Farmacêuticas, v. 40, n. 3, p. 289-299, 2004.

CFBio. Conselho Federal de Biologia. Resolução no 2, de 5 de março de 2002. Aprova o Código de Ética do Profissional Biólogo. Publicado no DOU, Seção 1, de 21.3.2002. Disponível em: $<$ https://crbio08.gov.br/biologo/codigo-de-etica/>. Acesso em 6 de jun. 2019.

CONCEA. Normativas do CONCEA para produção, manutenção ou utilização de animais em atividades de ensino ou pesquisa científica. Lei, decreto, portarias, resoluções normativas e orientações técnicas. 3a ed. 2016. Disponível em:

https://www.mctic.gov.br/mctic/export/sites/institucional/institucional/arquivos/concea/240230.pd f>. Acesso em 29 de out. 2018.

CFMV. Conselho Federal de Medicina Veterinária. Resolução N. 879, de 15 de fevereiro de 2008. Disponível em:<http://portal.cfmv.gov.br/lei/index/id/330>. Acesso em 6 de jun. 2019.

COSTA-NETO, E.M. Zooterapia popular no Estado da Bahia: registro de novas espécies animais utilizadas como recursos medicinais. Ciência \& Saúde Coletiva, v. 16, n. Supl 1, p. 1639-1650, 2011.

CROOK, R. J.; WALTERS, E.T. Nociceptive behavior and physiology of molluscs: animal welfare implications. Ilar Journal, 52(2), p.185-195, 2011.

DECLARAÇÃO UNIVERSAL DOS DIREITOS DO ANIMAL. Princípios éticos na experimentação animal. 1978. Disponível em: <http://portal.cfmv.gov.br/uploads/direitos.pdf > . Acesso em 28 de abr. 2019.

DEMO, P. Metodologia Científica em Ciências Sociais. São Paulo: Atlas, 2002. 
DINIZ, R. et al. Animais em aulas práticas: podemos substituí-los com a mesma qualidade de ensino? Revista Brasileira de Educação Médica, v. 2, n.30, p. 31-41, 2006.

ELWOOD, R.W. Pain and suffering in invertebrates? Ilar Journal, v. 52, n.2, p. 175-184, 2011.

FEIJÓ, A.M.G. et al. A. Análise de indicadores éticos do uso de animais na investigação científica e no ensino em uma amostra universitária da Área da Saúde e das Ciências Biológicas. Scientia Medica, v. 18, n. 1, p. 10-19, 2008.

FEIJÓ, A.M..G; MACEDO-BRAGA, L.M.G.; PITREZ, P.M. C. Animais na pesquisa e no ensino: aspectos éticos e técnicos. Porto Alegre: EDIPUCRS, 2010.

FISCHER, M. L. Ética no uso de animais em atividades científicas e acadêmicas (Coleção Ética em pesquisa Livro 3), Curitiba: PUCPRESS, 2017.

FISCHER, M.L. et al. Concepção, implementação e consolidação do comitê de ética no uso de Animais da PUCPR. Estudos Biologia, v. 361, p. 247-260, 2014.

FISCHER, M. L. et al. A percepção da dor como parâmetro de status moral em animais não humanos. Revista conexão ciência, v. 11, n.2, p. 31-41, 2016.

FISCHER, M.L., FURLAN, A.L.D. Metodologias ativas no ensino superior: é possível a substituição do uso de animais nas aulas práticas de zoologia? In: RAULI P. M. F. et al (orgs.). Bioética e Metodologias Ativas no ensino aprendizagem. Curitiba: CRV, 2018. pp. 175-187.

FISCHER, M.L., MOSER, A.M., DINIZ, A.L.F. Bioética e Educação: a utilização do nivelamento moral como balizador para construção de um agente moral consciente, autônomo e reflexivo. In: RENK, V. E. (org). Bioética Múltiplos Olhares. Curitiba: Prismas, 2016. p. 33-65.

FISCHER, M.L.; OLIVEIRA, G.M. Ética no uso de animais: a experiência do comitê de ética no uso de animais da Pontifícia Universidade Católica do Paraná. Estudos de Biologia, v. 34, n. 83, p. 1-12, 2012.

FISCHER, M.L.; SANTOS, J.Z. Bem-estar em Invertebrados: um parâmetro ético de responsabilidade científica e social da pesquisa?. Revista Latinoamericana de Bioética, v. 18, n. 34, p. 18-35, 2017.

FISCHER, M.L.; TAMIOSO, P.R. Perception and position of animals used in education and experimentation by students and teachers of different academic fields. Estudos de Biologia, v. 35, n. 84, 2013.

GREIFF, S. Alternativas ao uso de animais vivos na educação pela ciência responsável. São Paulo: Instituto Nina Rosa, 26p, 2003.

HÖTZEL, M.J.; MACHADO FILHO, L.C.P. Bem-estar animal na agricultura do século XXI. Revista de etologia, v. 6, n. 1, p. 3-15, 2004.

JUKES, N.; CHIUIA, M. From guine a pigtocomputer mouse. 2. ed. Leicester: Interniche, 520 p, 2003.

LEVAI, L.F. O direito à escusa de consciência na experimentação animal. Pensata Animal, 2010, n. 2, $12 \mathrm{p}$. 
LIMA, K. E., MAYER, M., CARNEIRO-LEÃO, A.M., VASCONCELOS, S.D. Conflito ou convergência? Percepções de professores e licenciandos sobre ética no uso de animais no ensino de zoologia. Investigações em Ensino de Ciências, v. 13, n.3, p. 353-369, 2008.

MARGALHÃES, M.; ORTÊNCIO-FILHO, H. Alternativas ao uso de animais como recurso didático. Arquivos de ciência de Veterinária de Zoologia da Unipar, v.9, p.147-154, 2006.

MATERA, J. M. et al. Nível de aprendizado nos métodos substitutivos ao uso de animais no ensino. Revista CFMV, v. 15, n. 46, p. 01-84, 2009.

MITRE, S. M. et al. Metodologias ativas de ensino-aprendizagem na formação profissional em saúde: debates atuais. Ciência \& saúde coletiva, v. 13, p. 2133-2144, 2008.

MORALES, M. M. Métodos alternativos à utilização de animais em pesquisa científica: mito ou realidade?. Ciência e Cultura, v. 60, n. 2, p. 33-36, 2008.

MORAN, J. Mudando a educação com metodologias ativas. Coleção Mídias Contemporâneas.

Convergências Midiáticas, Educação e Cidadania: aproximações jovens, v. 2, p. 15-33, 2015.

MOURA-LEITE, J. C.; FISCHER, M.L. Questões éticas em pesquisas conduzidas com animais silvestres na natureza, no laboratório e em cativeiro. Curitiba: PUCPRESS, 2018.

PASSERINO, A.S.M. et al. Aulas práticas com animais vivos. Estudos de Biologia, , v. 36, p.1-12, 2014.

POTTER, V.R. Bioética, ponte para o futuro. Trad. Diego Carlos Zanella. São Paulo: Edições Loyola, 2016

REIS, P.C.; TRÉZ, T.A. A experimentação animal na Universidade Federal de Goiás: elementos para uma abordagem crítica. Revista Contrapontos, v. 9, n. 2, p. 77-89, 2009.

REGAN, T. Jaulas vazias: encarando o desafio dos direitos animais. Trad.: Regina Rheda. Porto Alegre: Lugano, 2006.

RIVERA, E. A. B. Ética na experimentação animal e alternativas ao uso de animais em pesquisas e testes. In: Rivera EAB, Amaral MH, Nascimento VP, editores. Ética e bioética aplicadas à medicina veterinária. Goiânia: Gráfica da UFG, 2006. p. 159-199.

RODRIGUES, C. F. Liminar em mandado de segurança: Lei 12.016/2009. Revista de processo, 2010, v. 35, n. 190, p. 197-209.

RUSSEL, W. M. S.; BURCH, R. L. The Principles of Humane Experimental Technique. England: Universities Federation for Animal Welfare, 1959.

SCHANAIDER, A.; SILVA, P. C. Uso de animais em cirurgia experimental. Acta Cir. Bras, v. 19, n. 4, p. 441-447, 2004.

SEIXAS, M. M. et al. Consciência na substituição do uso de animais no ensino: aspectos históricos, éticos e de legislação. Revista Brasileira de Direito Animal, v. 5, n. 6, p. 71-96, 2014.

TRZ, T. A. Experimentando a desumanização: Paulo Freire e o uso didático de animais. Revista Brasileira de Ensino de Ciência e Tecnologia, v. 4, n. 2, p. 50-66, 2011. 
TRÉZ, T. A. Considerações sobre o conceito dos 3Rs e o potencial conflito com novas compreensões do animal experimental. Revista Brasileira de Zoociências, v. 19, n. 2, p. 97-113, 2018.

TUDURY, E. A.; POTIER, G.M.A. Métodos substitutivos ao uso de animais vivos no ensino-métodos alternativos para aprendizado prático da disciplina técnica cirúrgica veterinária. Ciência Veterinária nos Trópicos, v. 11, p. 92-95, 2008.

VALERIO, P. M. Espelho da Ciência, Série Especial. FINEP/IBICT, 2005.

VARGAS, J. M. S. B.; CERVI, T.M.D. Direito dos animais não humanos versus cultura acadêmica: a experimentação científica em questão. RIDB, n. 1, v. 42, p. 449-472, 2012.

VIEIRA, S.; HOSSNE, W. S. Experimentação com seres humanos. Ciência \& Ensino, 2006, v. 2, n. 1, p. 7-9.

VILLIERS, R.; SOMMERVILLE, J. Prospective biology teachers \attitudes toward animal dissection: implications and recommendations for the teaching of biology. South African Journal of Education, v. 25, n. 4, p. 247-252, 2005.

Submetido: $31 / 10 / 2019$

Aprovado: $31 / 03 / 2020$ 